\title{
Targeted Myocardial Restoration with Injectable Hydrogels - In Search of The Holy Grail in Regenerating Damaged Heart Tissue
}

\author{
Faizus Sazzad ${ }^{1 \dagger}$, Michał Kuzemczak ${ }^{2,3 \dagger}$, Engracia Loh ${ }^{1}$, Wellington $W^{1}{ }^{1}$ and Theo Kofidis $1,4 *$ \\ 1 Myocardial Experimental Laboratory, Department of Surgery, Yong Loo Lin School of Medicine, National \\ University of Singapore, Singapore; surmfs@nus.edu.sg (F.S.); surtk@nus.edu.sg (T.K.); \\ engracia_loh@u.nus.edu (E.L.); wellingwu118@gmail.com (W.W.) \\ 2 Department of Invasive Cardiology, Central Clinical Hospital of the Ministry of Interior and Administra- \\ tion, Warsaw, Poland; michal.kuzemczak@gmail.com (M.K.) \\ 3 Poznan University of Medical Sciences, Poznań, Poland \\ 4 Department of Cardiac, Thoracic and Vascular Surgery, National University Health System, Singapore; \\ * Correspondence: surtk@nus.edu.sg; Tel.: +65 67726505 \\ † These authors contributed equally.
}

\begin{abstract}
The peril of a 3-dimensional, robust and sustained myocardial restoration by means of Tissue Engineering is that it still remains a largely experimental approach. Prolific protocols have been developed and tested in small and large animals, but as clinical cardiac surgeons, we have not come to the privilege of utilizing any of them in our clinical practice. The question arises: why? The heart is a unique organ, anatomically and functionally. It is not an easy target to replicate with current techniques, or even to support its viability and function. Currently available therapies fail to reverse the loss of functional cardiac tissue, the fundamental pathology remains unaddressed and a heart transplantation is an ultima ratio treatment option. Owing to equivocal results of cell-based therapies, several strategies have been pursued to overcome limitations of the current treatment options. Preclinical data as well as first-in-human studies conducted to date have provided important insights into the understanding of injection-based approaches for myocardial restoration. In the light of the available data, injectable biomaterials suitable for transcatheteter delivery appear to have the highest translational potential,. This article presents a current state-of-the-art in the field of hydrogel-based myocardial restoration therapy.
\end{abstract}

Keywords: Hydrogel; Extracellular matrix hydrogels; Myocardial infarctions; Myocardial infarction therapy; Cardiac stem cell therapy; Tissue engineering; Cell-based therapy.

\section{Introduction}

"Stem cells are the future of heart treatment;... and they will always be" Norman Shumway

This may constitute a somewhat nihilistic approach, by mouth of an authority in Cardiac Surgery and heart failure treatment, yet holds more or less true to this day, simply taken from the angle of clinical implementation, in form of a comprehensive, recommended, if not guideline supported protocol. Twenty five years into myocardial restoration attempts following myocardial injury, there isn't a single efficient, robust and sustained impact on the injured heart muscle following ischemic insult. Approaches so far have been encompassing various types of cells, cell products or derivatives, scaffolds of various physical conditions, as well as multiple administration routes. It would be beyond the scope of the present paper to revisit them all, but, in brief, they all hold promise and peril.

The unique and complex structure of healthy and injured myocardium 
Gerard Buckberg, with his seminal paper "The Helix and the Heart" has awaken many aspired myocardial restorers to the fact that the heart is not a quiescent, homogenously built target, but rather a highly asymmetric, anisotropic and angiotropic organ, featuring an intricate architecture [1]. Not a spot in the heart is built like the other. The heart muscle is not a continuous layer but rather 3 layers superimposed at any given point, which can be folded and unfolded like a ribbon, as demonstrated by Buckberg [1]. This leads to a systematic overlay of 3 layers at any given spot, and the formation of critical intercalations and physical sheer stresses, which are organized in an optimal fashion to A: form an oval shaped vortex, and B: maximize contractile force at the best energy economy. This explains why a systolic diameter increase of only around $8 \%$ at the myofiber level translates in a much more disproportionately high fractional shortening, Ejection Fraction and left ventricular wall thickening [2] (Figure 1, B). The arrangement of myofibers, their communications and intercalations, the electrical signal propagation, the fibrous skeleton, the arrangement of supplying arterioles, all render the myocardium a very anisotropic target, where random injection of cells of any kind remains rather unimpressive, in terms of real health gain and symptomatic relief, from a clinician's point of view.

The Vicious Circle of Myocardial Ischemia and the mechanics of Remodelling

$\mathrm{Vu}$ et al. had postulated that the acute myocardial injury known as infarct triggers a cascade of events with severe cellular and functional impact [2]. This vicious circle is selfperpetuating, resulting in the so called "non ischemic expansion of the infarct", unrelated and not dependent on further coronary occlusions (Figure 1, A). This is largely due to mechanical shift of the myocardial plates, and a series of biological phenomena with architectural sequelae. When acute myocardial ischemia and injury manifest, cell death ensues. Enzymatic damage to the tissue is next, with the release of so called "danger signals" (derivatives of purine metabolism, free radicals etc) cause macrophagy and apoptosis [3]. This perpetuates the cell death cycle, stimulation of remodelling mechanisms which result to a scar formation. As a result, the affected myocardium thins out, while the surrounding myocardium may become temporarily dysfunctional as well. When the LV wall thins out, the modified Laplace law [4] of the oval of the heart takes effect, thus leading to extreme circumferential wall stress, more cell death [5] and architectural remodelling [2], and drop of contractility and Ejection Fraction [1,2] (Figure 1, C), as compared to that in the heart of a healthy individual [6] (Figure 1, B). The outcome is proportional to the extent of tissue loss and dysfunction and may encompass multiple segments of the LV, best to be captured by nuclear scans and MRI.
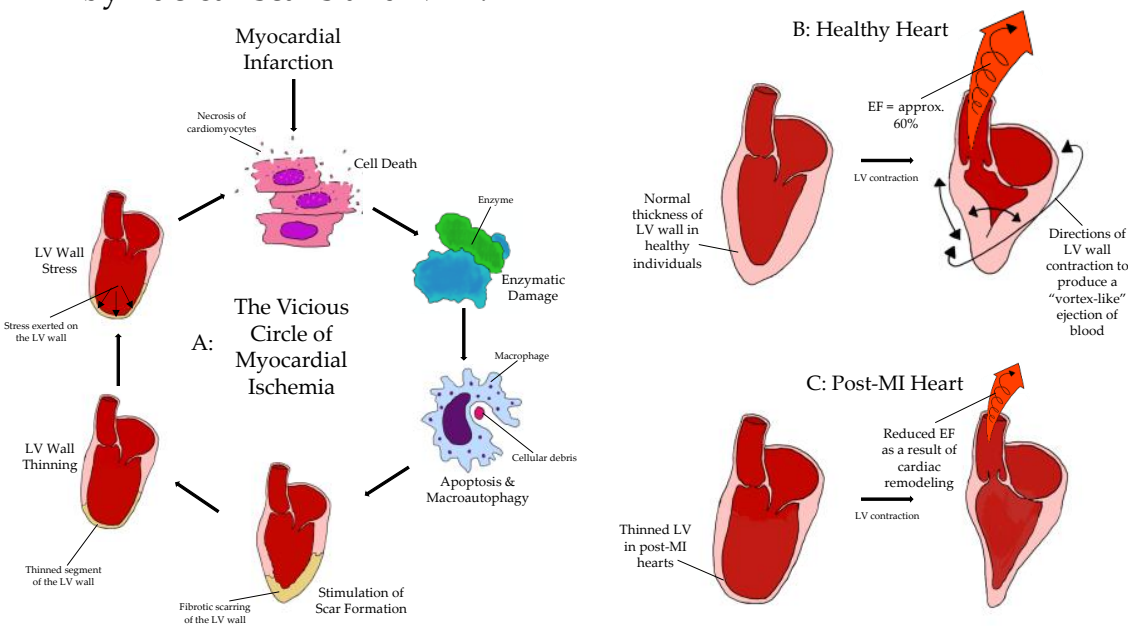

Figure 1. Vicious Circle of Myocardial Ischemia and ventricular wall remodeling after MI. EF: ejection fraction. A: Representative diagram of the "vicious circle" of myocardial ischemia, kickstarted by an initial myocardial infarction. B: Contraction of the LV in a healthy heart, and the EF produced as a result of efficient contraction. C: Contraction of a post-MI heart and the reduced EF produced as a result of altered cardiac architecture. 
Cell-based therapy - unfulfilled hopes or misguided expectations? Why not only cells?

The prevailing dogma suggesting that adult mammalian cardiomyocytes are postmitotic cells with no ability to renewal has been recently overthrown by studies demonstrating a low-level proliferation, even in adult hearts [7]. However, the regenerative capacity is minimal and insufficient to overome the loss of cardiac cells following MI. The inability of the adult heart to regenerate has yielded several preclinical and clinical studies focused on different cell-based therapies. Despite very promising preclinical results, so far these have not been translated into clinical practice. One of the major challenges limiting their clinical application are low retention and survival rates, very limited trans-differentiation into cardiomyocytes, safety and, in some cases, ethical concerns.

Over the last few decades, cell therapy has been applied in clinical myocardial restoration. Though the result is non-conclusive, some studies have shown the attenuation of the ventricular remodelling. The ensuing hostile and inflammatory environment results in rapid death of injected cells, or lack of integration thereof. It is incomprehensive, and in the vast majority of studies proven that injected cells do not organize in an integrated syncytium, which excites orchestrated contractility. Depending on the type of cells randomly injected, different complications occur [8]. Solid scaffolds, even if adding thickness to the aneurysmatic scar, have not proven themselves as viable solution either, particularly due to the necessity for open heart surgery to implant them (Figure 2).

A

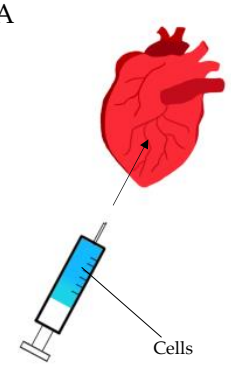

B

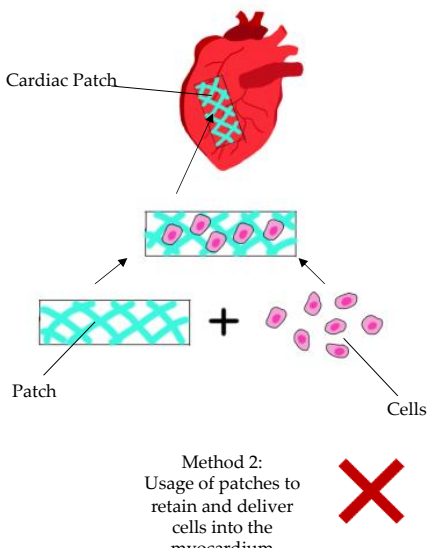

C

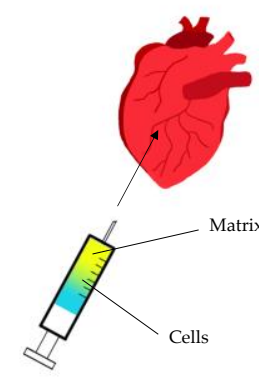

Method 3:

Intramyocardial delivery of cells
retained in a viscous

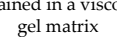

Figure 2. Diagram showing the current methods of regenerative cardiac therapy. A: Method 1 - Intramyocardial delivery (injection) of stem cells only, without a retaining matrix. B: Method 2 - Seeding of cells into a patch like matrix, which is then implanted onto the epicardium via sutures or glue. C: Method 3 - Intramyocardial injection of cells / active ingredient retained in a gel matrix either during an open surgery (thoracotomy) or in a minimal invasive manner (percutaneously etc.).

There is obvious need for a targeted, less invasive myocardial restoration treatment, which does not add too much stand-alone trauma to the patient and can be integrated in a viable clinical protocol, to be adopted by Cardiologists as well. Arising from the above pain-points, we have long shifted our focus from stem cells to liquid compounds, with following key value propositions:

- Injectable, hence minimally invasive, administration

- Autologous material, not of stem cell nature, to be derived simply, during treatment

- Polytherapy approach to address concomitant aspects of the vicious circle of myocardial ischemia (Antioxidants, Purine Metabolism blockers/Anti-inflammatory drugs)

- $\quad$ Easy adoption and clinical penetration in the horizon

\section{Materials and Methods}


A literature search was performed using the Preferred Reporting Items for Systematic Reviews and MetaAnalyses (PRISMA) guidelines [9] electronically. We conducted records scrutiny on Medline (via PubMed), Embase, and Web of Science from inception to 31st March 2021. A repetitive and exhaustive combination of the following 'Medical Subject Headings' (MeSH) were used: "Medical Subject Headings" (MeSH) terms were used: 'Hydrogels', 'Extracellular matrix hydrogels', 'Tissue engineering', 'Myocardial infarctions', 'Myocardial infarction therapy', 'Cardiac stem cell therapy', 'Cell-based therapy'. The full search strategy can be found in the supplementary materials (Supplementary Figure S1). Relevant articles were screened and systematically assessed with inclusion and exclusion criteria applied.

The inclusion criteria included any experimental cohort studies in which large animals or patients underwent injectable delivery of hydrogel and/or hydrogel compound analogure for its effect analysis on ischemic heart disease. Furthermore, only studies published after the year 2000 were included to prevent using outdated data. Articles with hydrogel compund processing (lab experiment) and in-vitro experiments, small animal studies and case reports were excluded. Additionally, any studies that were not written in the English language were excluded. Three authors (E.L., W.W. and F.S.) independently abstracted details of the study characteristics, the myocardial infarct (MI) creation and hydrogel characteristics and delivery method, and the outcomes measured. Data extracted with respect to the infarct creation and hydrogel characteristics and delivery method included: method of MI creation, artery involved, cell delivered via hydrogel or its analogues, type of matrix, method of delivery to myocardium. Data extracted with respect to the outcomes measured included: any data related to functional and morphological outcomes of the heart. Outcomes were then grouped according to the modality they were measured with. All outcomes are expressed as the treatment group outcome when compared to the control group.

\section{Findings}

The systematic search revealed a total of 28,704 papers. After 13,775 duplicates were excluded, 14,929 papers remained for screening. Based on the title and abstract, irrelevant articles were excluded, leaving 70 papers for full-text review. 61 out of these 70 papers could be retrieved. Following a full-text review of these papers, a final 19 papers were included. 2 papers were added to the final pool via additional sources, leaving a total of 21 papers for inclusion into the present study. The characteristics of the study population are summarised in Table 1. Experimental grouping and aim of the included study of 613 large animals and 15 human subjects has been plotted. The study characteristics did not differ markedly in thier aim but, diversification observes in grouping and use of animal subjects. In studies including Zhou, D et al. [15] and Liu, Y et al. [24] the recipients age and sex were not categorised. Hydrogel characterizaton and its mode of delivery is tabulated in Table 2. Few studies reported the delivery of injectable hydrogel without a celluar componant $[11,12,17,21,26,27,30]$ while the rest chose different composition of cells and matrix.

\subsection{Treatment with hydrogel improves systolic and diastolic cardiac function}

Out of the 21 studies, 17 measured systolic function via LVEF, and 4 via SV (Table 2). Yamamoto, T et al. 2001 [14], Chang, M.Y et al. [20,25], Li Y.D et al. [23] also measured diastolic function via LV EDP. All the studies that measured LVEF reported an increase, with the exception of Yamamoto, T et al. [14], which reported an equivocal outcome. These parameters were frequently measured via 3 main modalities: echocardiography, magnetic resonance imaging (MRI), or ventricular catheterization. Out of the 21 studies, only Leor, J et al. [11] did not measure functional outcomes of the treatment group.

Table 1. Characteristics of the included studies 


\begin{tabular}{llllll}
\hline Author & Species & Sex/Age & $\begin{array}{l}\text { Weight } \\
(\mathbf{k g})\end{array}$ & Number Grouping & Aim of the study \\
$\begin{array}{l}\text { Giordano, C et } \\
\text { al. 2013 [10] }\end{array}$ & $\begin{array}{l}\text { Swine, }{ }_{\text {Y }} \text { Forkshire Pig } \\
\text { F/- }\end{array}$ & $8-10$ & 32 & $\begin{array}{l}\text { Control: 10; CAC: 8; } \\
\text { CAC+Matrix: 7; Death=7 }\end{array}$ & $\begin{array}{l}\text { To investigate the effects of biopolymer- } \\
\text { supported delivery of circulating }\end{array}$
\end{tabular}

angiogenic cells.

\begin{tabular}{|c|c|c|c|c|}
\hline $\begin{array}{l}\text { Leor, J et al. } 2009 \\
\text { [11] }\end{array}$ & $\begin{array}{l}\text { Swine, } \\
\text { Domestic }\end{array}$ & $50-60 \quad 58$ & $\begin{array}{l}\text { Death=22; Exclusion=1; } \\
\text { Control=16; } \\
\text { Intervention=19 }\end{array}$ & $\begin{array}{l}\text { To investigate if a selective intracoronary } \\
\text { injection of alginate solution will result in } \\
\text { localised gelation as scaffold in the infarcted }\end{array}$ \\
\hline
\end{tabular}

\begin{tabular}{lllll}
\hline $\begin{array}{l}\text { Matsumura, Y et Swine, } \\
\text { al. } 2019 \text { [12] }\end{array} \quad$ Forkshire Pig months & $20-30$ & 12 & Control: 6; Treatment: 6
\end{tabular}
$\begin{array}{ll}\text { al. } 2019 \text { [12] } & \text { Yorkshire Pig months }\end{array}$ tissue.

\begin{tabular}{|c|c|c|c|c|c|}
\hline $\begin{array}{l}\text { Qiang Wang et } \\
\text { al. 2021 [13] }\end{array}$ & $\begin{array}{l}\text { Chinese } \\
\text { Pama } \\
\text { Minipig }\end{array}$ & $\begin{array}{l}\mathrm{M} / 6 \\
\text { months }\end{array}$ & $15-20$ & $\begin{array}{l}\text { Control: 15; hUMSC: } 15 ; \\
\text { hUMSC with Collagen: } 15\end{array}$ & $\begin{array}{l}\text { To investigate if an injectable collagen } \\
\text { scaffold promotes the long-term retention of } \\
\text { transplanted stem cells. }\end{array}$ \\
\hline $\begin{array}{l}\text { Yamamoto, T et } \\
\text { al. } 2001 \text { [14] }\end{array}$ & $\begin{array}{l}\text { Canine, } \\
\text { Mongrel }\end{array}$ & -/Adult & $20.3 \pm 0.628$ & $\begin{array}{l}\text { AGHM-bFGF: } 13 \text {; bGFG } \\
\text { only: 9; AGHM only: } 6\end{array}$ & $\begin{array}{l}\text { To find out if bFGF-impregnated AGHM } \\
\text { would enhance collateral development to } \\
\text { the infarct area. }\end{array}$ \\
\hline $\begin{array}{l}\text { Zhou, D et al. } \\
2012 \text { [15] }\end{array}$ & $\begin{array}{l}\text { Canine, } \\
\text { Mongrel }\end{array}$ & $-/-$ & $9-14$ & $\begin{array}{l}\text { MI: 5; MI + NS: 6; CH: 6; } \\
\text { CH + GF: 7; Death=8 }\end{array}$ & $\begin{array}{l}\text { Whether TMJR with chitosan scaffolds } \\
\text { retains channel patency and enhance } \\
\text { angiogenesis. }\end{array}$ \\
\hline
\end{tabular}

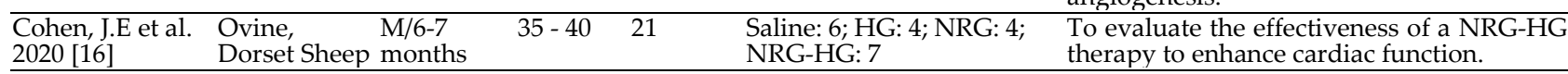

\begin{tabular}{lllll}
\hline Contessotto, P et Ovine, & M/8 & $30-40$ & 39 & MI only (7 day): 6; MI only To evaluate the effectiveness of an ECM-
\end{tabular}

$\begin{array}{lll}\text { al. 2021 [17] } & \text { Romanov months } & \text { (28 day): 8; PBS: 6; ELRs- mimicking hydrogel in modulating post- } \\ & \text { Sheep } & \text { Hydrogel: 6; Death: 12; }\end{array}$

\begin{tabular}{llll}
\hline Li, Y et al. 2021 & Swine, \\
{$[18]$} & Yucatan mini
\end{tabular}
Hydrogel: 6; Death: 12; ischemic.

Sham: 3; Saline: 5; agomiR- To demonstrate that a microRNA-21-5p 21-5p: 5; Gel@MSN/miR- delivery system enables both immuno NC: 6; Gel@MSN/miR-21- modification and enhanced angiogenesis pigs 5p: 6 for myocardial infarction

\begin{tabular}{llll}
\hline Purcell, B.P et al. $\begin{array}{l}\text { Swine, } \\
\text { Yorkshire Pig }\end{array}$ M/- & 20 & 26 & Sham:5; MI/Saline: 7; \\
& & & MI/HAMMPS: 7; \\
& & & MI/HAMMPS/rTIMP-3: 7
\end{tabular}
To investigate if the localised delivery of a MMP-sensitive biomaterial that releases a MI/HAMMPS/rTIMP-3: 7 recombinant TIMP holds promise as a means to interrupt adverse post-MI remodeling

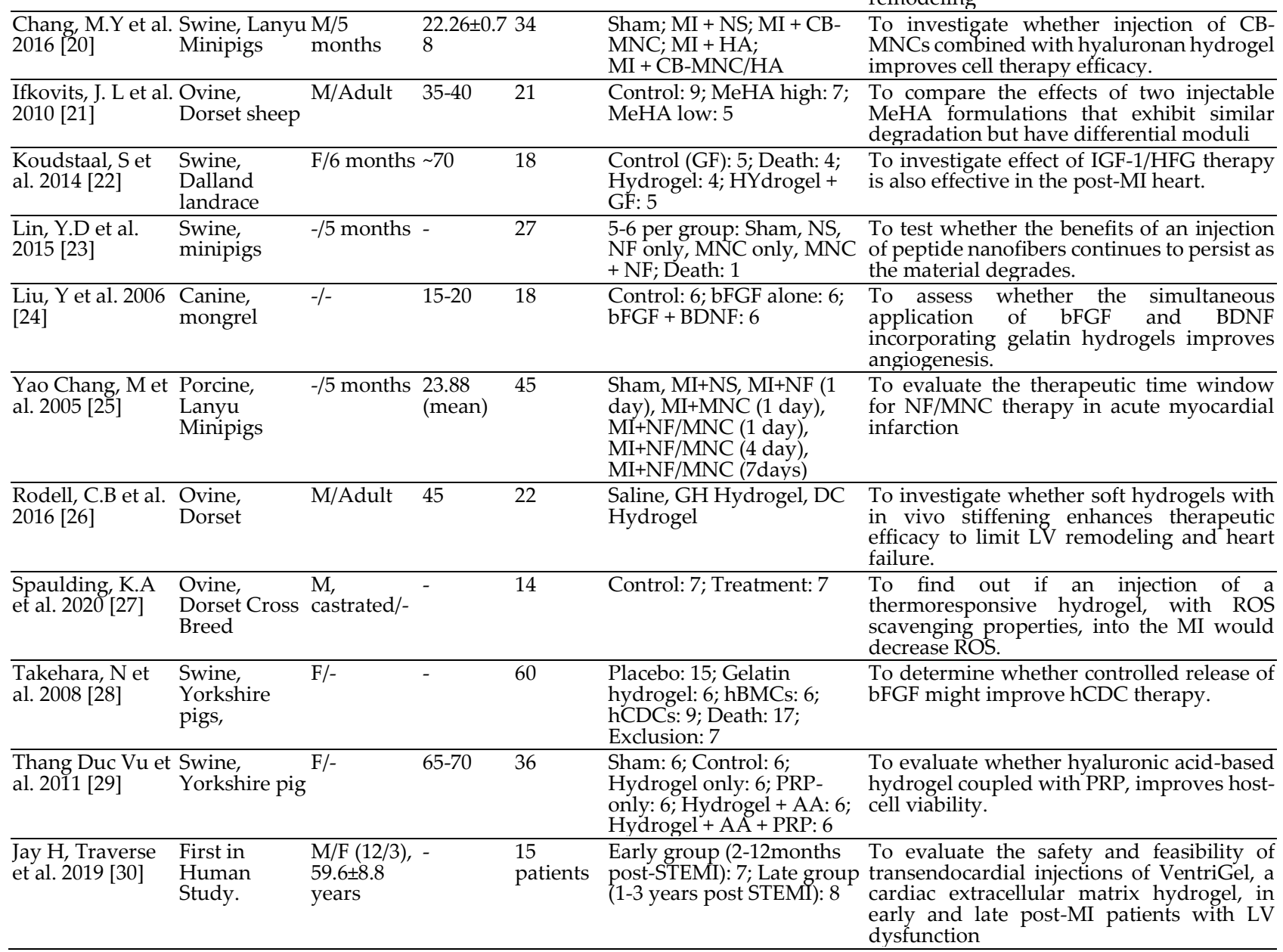


Ventricle size was frequently measured via ESV, EDV, wall thickness and LV mass. The common modalities used to measure these parameters included: echocardiography, MRI, and immunohistochemistry. Out of the 21 studies, Giordano, C et al. 2013 [10], Yamamoto, T. et al. 2001 [13], Zhou, D et al. 2012 [15], Liu, Y et al. 2006 [24] and Takehara, $\mathrm{N}$ et al. 2008 [28] did not measure the direct effect of treatment on LV remodeling. The remaining 16 studies that looked into LV remodeling reported either attenuated or equivocal LV remodeling. This would suggest that injectable hydrogels have the ability to retain the highly complex and intricate architecture of the heart, post-MI, resulting in increased EF [2]. This would be in line with the findings of studies [12,13,16-23,25,26,27,29,30], which reported both attenuated LV remodeling and an increase in EF.

\subsection{Treatment with hydrogel reduces cardiac fibrosis}

Cardiac fibrosis was frequently quantified via scar size and the extent of fibrosis. These parameters were measured via immunohistochemistry, Masson's trichrome stain, MRI and computed tomography (CT). Out of the 21 studies, only 5 studies $[12,15,17,18$, 25] measured the effect of treatment on fibrosis, with Zhou, D et al. [15] reporting equivocal scar size, and the rest reporting reduced fibrosis with treatment.

\subsection{Treatment with hydrogel supports angiogenesis post-infarction}

The degree of angiogenesis was mainly quantified using blood vessel density via immunohistochemistry staining. Out of the 21 studies, only $11[10,14,15,17,18,20,22-25,29]$ measured blood vessel density, all of which reported an improved effect, implying that hydrogel treatment can have a positive effect on angiogenesis post-MI. Zhou, D et al [15] particularly focuses on the density of specific blood vessels, namely arterioles, small vessels and larger arterioles, all of which show an increase in density.

\section{Discussion}

\subsection{Post-MI survivability}

In the vast majority of cases, $\mathrm{MI}$ is a consequence of a vulnerable plaque rupture and a subsequent intracoronary thrombosis. The process initiates maladaptive changes in myocardium termed "cardiac remodelling" which may result in the development of HF (Figure 2). The clinical sequelae are encountered in up to three-quarters of patients within 5 years after their first coronary event [31]. Importantly, HF has not only a significant impact on patients' functional capacity and quality of life, but the disease also significantly affects their life expectancy. Available data indicates that approximately half of patients with HF do not survive more than 5 years after the diagnosis [32], meaning that despite advances in cardiac care, survival rates in this patient population are still very poor and comparable to those observed in many types of cancer [33,34]. Given the above, more still needs to be done to tackle the burden of the disease more efficiently, thus, triggering alternative mono- or poly-therapeutic treatments using viable matter and scaffolds.

\subsection{Injectable hydrogel-based approach for cardiac tissue engineering}

Owing to the intricate myocardial architecture and function, we believe that the triple approach, i.e. enhancing viability, counteracting inflammation and stabilizing the diminishing architectural integrity of the Left Ventricle yields the best restorative effect. One of the most promising therapeutic compounds are hydrogel-based biomaterials that can provide not only a mechanical support for a failing heart, but also can serve as a vehicle for cells, growth factors and drugs. Beacause of their potential for minimally invasive transcatheter delivery, injectable hydrogels appear to be one of the most promising ones in terms of their potential clinical application. Several types of hydrogel- 


\begin{tabular}{|c|c|c|c|c|c|c|c|c|}
\hline Author & Echo & MRI & $\mathrm{CT}$ & PET & Ventricular catheterization & Immunohistochemistry & $\begin{array}{l}\text { Masson's } \\
\text { Trichrome } \\
\text { Staining }\end{array}$ & Other \\
\hline $\begin{array}{l}\text { Giordano, C et al. } \\
2013 \text { [10] }\end{array}$ & $\begin{array}{l}\text { LVEF } \uparrow \\
\text { WMSI } \downarrow\end{array}$ & & & $\begin{array}{l}\text { MBF during: Rest } \\
\downarrow \text { Stress } \uparrow, \text { MFR } \uparrow\end{array}$ & & BV amount $\uparrow$ & & \\
\hline $\begin{array}{l}\text { Leor, J et al. } 2009 \\
{[11]}\end{array}$ & $\begin{array}{l}\text { ES area } \downarrow, \mathrm{LV} \\
\text { mass } \downarrow\end{array}$ & & & & & Wall thickness $\uparrow$ & & \\
\hline $\begin{array}{l}\text { Matsumura, Y et } \\
\text { al. } 2019 \text { [12] }\end{array}$ & & 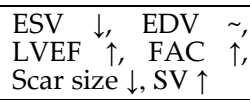 & & & & Angiotensin II $\uparrow$ & Cardiac fibrosis $\downarrow$ & $\begin{array}{l}\text { Stiffness } \uparrow(\text { Biaxial } \\
\text { mechanical) }\end{array}$ \\
\hline $\begin{array}{l}\text { Qiang Wang et al. } \\
2021 \text { [13] }\end{array}$ & $\begin{array}{l}\mathrm{LVEF} \uparrow \\
\mathrm{CO} \uparrow, \mathrm{SV} \uparrow, \\
\mathrm{ESV} \downarrow, \mathrm{EDV} \downarrow\end{array}$ & $\begin{array}{l}\mathrm{LVEF} \uparrow \\
\mathrm{CO} \uparrow, \mathrm{SV} \uparrow \\
\mathrm{ESV} \downarrow, \mathrm{EDV} \downarrow\end{array}$ & $\begin{array}{l}\text { Scar size: } \downarrow \\
\text { Infarct size: } \downarrow \\
\text { LVMV } \downarrow\end{array}$ & & & $\begin{array}{l}\text { Cell retention } \uparrow, \text { Arteriole } \\
\text { density } \uparrow \text {, Island-/strip- shaped } \\
\text { cTnT-positive cells } \uparrow\end{array}$ & & \\
\hline $\begin{array}{l}\text { Yamamoto, T et al. } \\
2001[14]\end{array}$ & & & & & $\begin{array}{l}\text { LVEF } \sim \text {, LVEDP , Antegrade } \\
\text { flow } \uparrow, \text { Wall motion } \sim \text { MBF in } \\
\text { ischemic region } \uparrow\end{array}$ & BV density $\uparrow$ & & \\
\hline $\begin{array}{l}\text { Zhou, D et al. } 2012 \\
\text { [15] }\end{array}$ & & & & & & $\begin{array}{l}\text { Endothelization } \uparrow \text {, Arteriole } \\
\text { and small vessel density } \uparrow, \\
\text { Larger arteriole density } \uparrow\end{array}$ & Cardiac fibrosis & $\begin{array}{l}\text { Size of infarct region: (via } \\
\text { weighing) }\end{array}$ \\
\hline $\begin{array}{l}\text { Cohen, J.E et al. } \\
2020[16]\end{array}$ & & & & & $\begin{array}{l}\text { LVEF } \uparrow, \text { Mean arterial pressure } \\
\uparrow \\
\text { EDV } \downarrow, \text { ESV } \downarrow, \text { ESPVR } \uparrow\end{array}$ & & & \\
\hline $\begin{array}{l}\text { Contessotto, P et } \\
\text { al. } 2021 \text { [17] }\end{array}$ & $\bar{L} \operatorname{LVEF} \uparrow$ & & & & & $\begin{array}{l}\text { Wall thickness } \uparrow \text {, Collagen } \\
\text { fibres } \downarrow \\
\text { BV density } \uparrow \text {, Cardiomyocyte } \\
\text { preservation } \uparrow\end{array}$ & Cardiac fibrosis $\downarrow$ & \\
\hline $\begin{array}{l}\text { Li, Y et al. } 2021 \\
{[18]}\end{array}$ & $\begin{array}{l}\text { LVEF } \uparrow \\
\mathrm{EDV} \downarrow, \mathrm{ESV} \downarrow \\
\mathrm{LV} \text { EDd } \uparrow\end{array}$ & & $\begin{array}{l}\text { Scar size: } \downarrow \\
\text { Wall thickness } \\
\uparrow\end{array}$ & & & $\begin{array}{l}\text { BV density } \uparrow, \text { BV volume } \uparrow, \\
\text { Infarct size } \downarrow \\
\text { Immunomodulatory effect } \downarrow\end{array}$ & Cardiac fibrosis $\downarrow$ & \\
\hline $\begin{array}{l}\text { Purcell, B.P et al. } \\
2013 \text { [19] }\end{array}$ & $\begin{array}{l}\text { LVEF } \uparrow, \text { EDV } \\
\downarrow, \text { ESV } \downarrow \text {, Wall } \\
\text { thickness } \uparrow, \text { LV } \\
\text { mass } \downarrow\end{array}$ & & & & & & & $\begin{array}{l}\text { Transcriptional activity of } \\
\text { myofibroblasts and } \\
\text { profibrotic pathways } \downarrow \\
\text { (mRNA profiling) }\end{array}$ \\
\hline $\begin{array}{l}\text { Chang, M.Y et al. } \\
2016 \text { [20] }\end{array}$ & $\begin{array}{l}\text { LVEF } \uparrow \\
\text { IVS thickness } \uparrow\end{array}$ & & & & $\begin{array}{l}+\mathrm{dp} / \mathrm{dt} \uparrow,-\mathrm{dp} / \mathrm{dt} \uparrow \\
\mathrm{LV} \text { EDP } \downarrow, \mathrm{EDV} \downarrow\end{array}$ & $\begin{array}{l}\text { Cell retention } \uparrow \\
\text { BV density } \uparrow \\
\text { EC differentiation } \uparrow\end{array}$ & & $\begin{array}{l}\text { Scar size } \downarrow \\
\text { Wall thickness } \uparrow \text { (Via gross } \\
\text { cross-section) }\end{array}$ \\
\hline $\begin{array}{l}\text { Ifkovits, J. L et al. } \\
2010 \text { [21] }\end{array}$ & $\begin{array}{l}\mathrm{LVEF} \uparrow, \mathrm{ESV} \uparrow, \\
\mathrm{EDV} \uparrow, \mathrm{CO} \uparrow\end{array}$ & & & & & $\begin{array}{l}\text { Wall thickness } \uparrow \\
\text { Infarct area } \downarrow\end{array}$ & & \\
\hline $\begin{array}{l}\text { Koudstaal, S et al. } \\
2014[22]\end{array}$ & $\begin{array}{l}\text { LVEF } \uparrow \\
\text { EDV } \uparrow, \text { ESV } \uparrow, \\
\text { FAS } \uparrow, \\
\text { PRSW } \uparrow\end{array}$ & & & & & $\begin{array}{l}\text { Cardiomyocyte hypertrophy } \downarrow \text {, } \\
\text { Cardiomyocyte proliferation } \uparrow, \\
\text { Fibrosis extent } \downarrow, \text { BV density } \uparrow, \\
\text { C-kit number } \uparrow\end{array}$ & & \\
\hline $\begin{array}{l}\text { Lin, Y.D et al. } 2015 \\
{[23]}\end{array}$ & $\begin{array}{l}\text { LVEF } \uparrow, \text { PSV } \uparrow, \\
\text { E/A ratio } \downarrow\end{array}$ & & & & $\begin{array}{l}\mathrm{SV} \uparrow, \mathrm{AE} \uparrow,+\mathrm{dP} / \mathrm{dt} \uparrow,-\mathrm{dP} / \mathrm{dt} \uparrow, \\
\mathrm{PRSW} \uparrow, \mathrm{Emax} \uparrow, \mathrm{T}, \downarrow, \mathrm{ESV} \downarrow \\
\mathrm{EDV} \downarrow, \mathrm{ESP} \uparrow, \mathrm{EDP} \downarrow\end{array}$ & BV density $\uparrow$ & & \\
\hline $\begin{array}{l}\text { Liu, Y et al. } 2006 \\
{[24]}\end{array}$ & & LVEF $\uparrow$ & & & $\mathrm{MBF} \uparrow$ & $\begin{array}{l}\text { BV density } \uparrow, \text { bFGF expression } \\
\uparrow \\
\text { BDNF expression } \uparrow, \\
\text { Distribution of bFGF and } \\
\text { BDNF positive cells } \sim\end{array}$ & & \\
\hline
\end{tabular}

Table 2. Summary of outcome assessment - improved cardiac function in treatment groups with hydroge 


\begin{tabular}{|c|c|c|c|c|c|c|}
\hline $\begin{array}{l}\text { Yao Chang, M et } \\
\text { al. } 2005 \text { [25] }\end{array}$ & LVEF $\uparrow$ & & $\begin{array}{l}\text { IVS thickness } \uparrow \\
\text { Systolic function } \uparrow \\
\text { EDP } \uparrow, \text { EDV } \uparrow, \text { Emax } \uparrow\end{array}$ & $\begin{array}{l}\text { Infarct size } \downarrow \text {, Infarct length } \\
\text { ratio } \downarrow \\
\text { BV density } \uparrow \text {, Blood flow } \uparrow\end{array}$ & Cardiac fibrosis $\downarrow$ & $\begin{array}{l}\text { Stem cell retention } \uparrow \\
\text { (Confocal microscopy with } \\
\text { Dil and DAPI staining) }\end{array}$ \\
\hline $\begin{array}{l}\text { Rodell, C.B et al. } \\
2016 \text { [26] }\end{array}$ & & $\begin{array}{l}\text { LVEF } \uparrow \\
\text { EDV, ESV } \downarrow \\
\text { LV wall thickness } \uparrow\end{array}$ & & & & $\begin{array}{l}\text { Myofiber stress reduction } \uparrow \\
\text { (Via FE simulation model, } \\
\text { dimensions of model } \\
\text { obtained via MRI) }\end{array}$ \\
\hline $\begin{array}{l}\text { Spaulding, K.A et } \\
\text { al. } 2020 \text { [27] }\end{array}$ & $\begin{array}{l}\text { LVEF } \uparrow(2 \mathrm{wk}), \\
\mathrm{LVEF} \downarrow(6 \mathrm{wk}), \\
\mathrm{EDV} \uparrow \quad \mathrm{ESV} \uparrow\end{array}$ & $\begin{array}{l}\text { LV wall thickness } \uparrow \\
\text { Demembranated } \\
\text { muscle force } \uparrow\end{array}$ & & $\begin{array}{l}\text { Levels of ROS in BZ } \downarrow \\
\text { FL-MMP-2 } \downarrow\end{array}$ & & $\begin{array}{l}\text { SV } \uparrow(2 \text { weeks }) \\
\text { SV } \downarrow(6 \text { weeks) } \\
\text { PCWP } \uparrow \text { (Via Swan Ganz) }\end{array}$ \\
\hline $\begin{array}{l}\text { Takehara, N et al. } \\
2008 \text { [28] }\end{array}$ & $\begin{array}{l}\text { LVEF } \uparrow, \text { RWM } \\
\uparrow \\
\text { Myocardial } \\
\text { perfusion } \uparrow \\
\text { Infarct size } \downarrow\end{array}$ & $\begin{array}{l}\text { Stem cell retention } \\
\uparrow\end{array}$ & & Myocyte conversion $\uparrow$ & & \\
\hline $\begin{array}{l}\text { Thang Duc Vu et } \\
\text { al. } 2011 \text { [29] }\end{array}$ & $\begin{array}{l}\text { LVEF } \uparrow \\
\text { FAC } \uparrow \\
\text { EDV } \downarrow\end{array}$ & $\begin{array}{l}\text { LV mass } \downarrow \\
\text { LV collagen area } \\
\text { fraction } \downarrow \\
\text { Scar size } \downarrow\end{array}$ & & $\begin{array}{l}\text { BV density } \uparrow \\
\text { BV amount } \uparrow\end{array}$ & & \\
\hline $\begin{array}{l}\text { Jay H, Traverse et } \\
\text { al. } 2019 \text { [30] }\end{array}$ & & $\begin{array}{l}\text { LVEF: } \sim, \text { EDV } \downarrow \text {, } \\
\text { ESV } \downarrow \\
\text { Scar size: } \sim \text {, Viable } \\
\text { mass } \uparrow\end{array}$ & & & & $\begin{array}{l}\text { BNP } \downarrow, 6 \text {-min walk test } \\
\text { distance } \uparrow \\
\text { NYHA class } \uparrow, \quad \text { MLWHFQ } \\
\text { score } \uparrow\end{array}$ \\
\hline
\end{tabular}

based approaches for cardiac tissue repair have been investigated to date. Each 2 category of hydrogels has its advantages and disadvantages that can influence their 3 potential clinical applicability. There are various types of hydrogels with different 4 properties based on their origin (natural/synthetic), various mechanisms of cross- 5 linking, etc.

Based on the best evidence, we have observed a diversity of compounds with none 7 of the compositions showing clear superiority (Table 3). There are a number of 8 studies using accelualr hydrogel by changing the matix composition, more focused 9 to investigate whether hydrogel characteristics i.e stiffening enhances therapeutic 10 efficacy to limit LV remodeling and heart failure [26]. Synthetic Hydrogel: Poly 11 (NIPAAm-co-HEMA-co-MAPLA) (Sigma-Aldrich, USA) was used in some studies 12 $[12,27]$, but Hyaluronic acid-based hydrogel was the choice in most cases. Cell Types 13 include skeletal myoblasts (SKMs), CMs, and other progenitor cells capable of 14 differentiation to CMs like embryonic stem cell (ESC), ESC-derived CMs (ESCCMs), 15 and mesenchymal stem cells (MSCs) with limited potentials was investigated. 
Human umbilical mesenchymal stem cells [hUMSC] are in new focus [13] whereas basic fibroblast growth factor (bFGF); acidic gelatin hydrogel microspheres (AGHM); vascular endothelial growth factor (VEGF) are in use with non-superiority to each other. Vu et al used hyaluronic acid-based hydrogel coupled with PRP, showed improved host-cell viability [29]; while Traverse et al. [30] reported First-in-Human Study with VentriGel ${ }^{\mathrm{TM}}$ (ECM from decellularized porcine myocardium) in patients with 1st STEMI treated by PCI within a period of post-interventional 60 days to 3 years, found MRI evidence of LV remodeling and clinical improvement of the study subgroup.

Table-3: Hydrogel characterization and mode of delivery

\begin{tabular}{|c|c|c|c|c|c|}
\hline Author & $\begin{array}{l}\text { Method of MI } \\
\text { creation }\end{array}$ & Artery involved & Cell delivered & Type of matrix & $\begin{array}{l}\text { Method of delivery } \\
\text { to myocardium }\end{array}$ \\
\hline $\begin{array}{l}\text { Giordano, C } \\
\text { et al. } 2013 \\
{[10]}\end{array}$ & $\begin{array}{l}\text { Left thoracotomy with } \\
\text { ameroid constrictor }\end{array}$ & Proximal LCx & CAC & $\begin{array}{l}\text { Type-I rat tail collagen cross linked } \\
\text { with glutaraldehyde (BD } \\
\text { Bioscience, Oakville, Canada) }\end{array}$ & $\begin{array}{l}\text { Open; } \\
\text { Intramyocardial }\end{array}$ \\
\hline $\begin{array}{l}\text { Leor, J et al. } \\
2009 \text { [11] }\end{array}$ & Balloon occlusion & Mid-LAD artery & Acellular & $\begin{array}{l}\text { Sodium alginate (VLVG, } \\
\text { NovaMatrix, } \\
\text { FMC Biopolymers, Drammen, } \\
\text { Norway) }\end{array}$ & Intracoronary \\
\hline $\begin{array}{l}\text { Matsumura, } \\
\text { Y et al. } 2019 \\
{[12]}\end{array}$ & $\begin{array}{l}\text { Left thoracotomy with } \\
\text { suture ligation }\end{array}$ & $\begin{array}{l}\text { Between 1st and } \\
\text { 2nd diagonal } \\
\text { branches }\end{array}$ & Acellular & $\begin{array}{l}\text { Synthetic Hydrogel: } \\
\text { Poly(NIPAAm-co-HEMA-co- } \\
\text { MAPLA) (Sigma-Aldrich, USA) }\end{array}$ & $\begin{array}{l}\text { Open; } \\
\text { Intramyocardial }\end{array}$ \\
\hline $\begin{array}{l}\text { Qiang Wang } \\
\text { et al. } 2021 \\
{[13]}\end{array}$ & $\begin{array}{l}\text { Left thoracotomy with } \\
\text { suture ligation }\end{array}$ & $\begin{array}{l}\text { LAD distal to } \\
\text { origin of } 2 \text { nd } \\
\text { branch }\end{array}$ & hUMSC & Bovine collagen & $\begin{array}{l}\text { Open; } \\
\text { Intramyocardial }\end{array}$ \\
\hline $\begin{array}{l}\text { Yamamoto, } \\
\text { T et al. } 2001 \\
{[14]}\end{array}$ & $\begin{array}{l}\text { Left thoracotomy with } \\
\text { suture ligation }\end{array}$ & $\begin{array}{l}\text { LAD between } 1 \text { st } \\
\text { and } 2 \text { nd diagonal } \\
\text { branches }\end{array}$ & bFGF & AGHM & $\begin{array}{l}\text { Open; Subepicardial } \\
\text { implantation }\end{array}$ \\
\hline $\begin{array}{l}\text { Zhou, D et } \\
\text { al. } 2012 \text { [15] }\end{array}$ & $\begin{array}{l}\text { Left thoracotomy with } \\
\text { suture ligation }\end{array}$ & $\begin{array}{l}\text { LAD below 1st } \\
\text { diagonal branch }\end{array}$ & VEGF165 & $\begin{array}{l}\text { Temperature-responsive Chitosan } \\
\text { hydrogel }\end{array}$ & $\begin{array}{l}\text { Open; } \\
\text { Transmyocardial jet } \\
\text { revascularization }\end{array}$ \\
\hline $\begin{array}{l}\text { Cohen, J.E et } \\
\text { al. } 2020[16]\end{array}$ & $\begin{array}{l}\text { Left thoracotomy with } \\
\text { suture ligation }\end{array}$ & $\begin{array}{l}\text { 2nd and 3rd } \\
\text { diagonal branches } \\
\text { of LAD }\end{array}$ & $\begin{array}{l}\text { NRG (R\&D Systems, } \\
\text { Minneapolis, MN, } \\
\text { USA) }\end{array}$ & $\begin{array}{l}\text { HEMA-HA based hydrogel } \\
\text { (Lifecore Biomedical Inc., Chaska, } \\
\text { MN, USA }\end{array}$ & $\begin{array}{l}\text { Open; } \\
\text { intramyocardial }\end{array}$ \\
\hline $\begin{array}{l}\text { Contessotto, } \\
\text { P et al. } 2021 \\
{[17]}\end{array}$ & $\begin{array}{l}\text { Left thoracotomy with } \\
\text { suture ligation }\end{array}$ & $\begin{array}{l}\text { LAD from 1st } \\
\text { diagonal branch, } \\
\text { moving distally till } \\
\text { apex }\end{array}$ & Acellular & ELRs hydrogel & $\begin{array}{l}\text { Open; } \\
\text { Intramyocardial }\end{array}$ \\
\hline $\begin{array}{l}\text { Li, Y et al. } \\
2021[18]\end{array}$ & $\begin{array}{l}\text { Left thoracotomy with } \\
\text { suture ligation }\end{array}$ & $\begin{array}{l}\text { 1st two obtuse } \\
\text { marginal arteries } \\
\text { of LCx }\end{array}$ & $\begin{array}{l}\text { MSN/miR-21-5p } \\
\text { complex }\end{array}$ & Injectable hydrogel matrix & $\begin{array}{l}\text { Open; } \\
\text { Intramyocardial }\end{array}$ \\
\hline $\begin{array}{l}\text { Purcell, B.P } \\
\text { et al. } 2013 \\
\text { [19] }\end{array}$ & $\begin{array}{l}\text { Left thoracotomy with } \\
\text { suture ligation }\end{array}$ & $\begin{array}{l}\text { 1st two obtuse } \\
\text { marginal arteries } \\
\text { of LCx }\end{array}$ & Full-length rTIMP-3 & $\begin{array}{l}\text { Hyaluronic acid-based hydrogel } \\
\text { with MMP }\end{array}$ & $\begin{array}{l}\text { Open; } \\
\text { Intramyocardial }\end{array}$ \\
\hline $\begin{array}{l}\text { Chang, M.Y } \\
\text { et al. } 2016 \\
\text { [20] }\end{array}$ & $\begin{array}{l}\text { Left thoracotomy with } \\
\text { suture ligation }\end{array}$ & Mid-LAD & CB-MNC & Hyaluronic acid hydrogel & $\begin{array}{l}\text { Open; } \\
\text { Intramyocardial }\end{array}$ \\
\hline $\begin{array}{l}\text { Ifkovits, J. L } \\
\text { et al. } 2010 \\
{[21]}\end{array}$ & $\begin{array}{l}\text { Left thoracotomy with } \\
\text { suture ligation }\end{array}$ & $\begin{array}{l}\text { LAD and 2nd } \\
\text { diagonal coronary } \\
\text { artery }\end{array}$ & Acellular & $\begin{array}{l}\text { Methacrylated hyaluronic acid } \\
\text { macromers (MeHA) hydrogel }\end{array}$ & $\begin{array}{l}\text { Open; } \\
\text { Intramyocardial }\end{array}$ \\
\hline $\begin{array}{l}\text { Koudstaal, S } \\
\text { et al. } 2014 \\
{[22]}\end{array}$ & $\begin{array}{l}75 \mathrm{~min} \text { intracoronary } \\
\text { balloon occlusion }\end{array}$ & LCx & IGF-1/HGF & UPy hydrogel & $\begin{array}{l}\text { Open; } \\
\text { Intramyocardial }\end{array}$ \\
\hline $\begin{array}{l}\text { Lin, Y.D et } \\
\text { al. } 2015 \text { [23] }\end{array}$ & $\begin{array}{l}\text { Left thoracotomy with } \\
\text { suture ligation }\end{array}$ & Mid-LAD & MNCs & Peptide nanofibers & $\begin{array}{l}\text { Open; } \\
\text { Intramyocardial }\end{array}$ \\
\hline $\begin{array}{l}\text { Liu, Y et al. } \\
2006 \text { [24] }\end{array}$ & $\begin{array}{l}\text { Left thoracotomy with } \\
\text { suture ligation }\end{array}$ & $\begin{array}{l}\text { LAD distal to 1st } \\
\text { diagonal branch }\end{array}$ & bFGF, BDNF & $\begin{array}{l}\text { Gelatin hydrogel (Boster } \\
\text { Bioengineering Company, Wuhan, } \\
\text { China) }\end{array}$ & $\begin{array}{l}\text { Open; } \\
\text { Intramyocardial }\end{array}$ \\
\hline $\begin{array}{l}\text { Yao Chang, } \\
\text { M et al. } 2005 \\
{[25]}\end{array}$ & $\begin{array}{l}\text { Left thoracotomy with } \\
\text { suture ligation }\end{array}$ & Mid-LAD & Bone marrow MNC & Peptide nanofibers & $\begin{array}{l}\text { Open; } \\
\text { Intramyocardial }\end{array}$ \\
\hline $\begin{array}{l}\text { Rodell, C.B } \\
\text { et al. } 2016 \\
\text { [26] }\end{array}$ & $\begin{array}{l}\text { Left thoracotomy with } \\
\text { suture ligation }\end{array}$ & $\begin{array}{l}\text { Selective ligation } \\
\text { of obtuse marginal } \\
\text { branches }\end{array}$ & Acellular & $\begin{array}{l}\text { Guest-host hydrogels; Dual- } \\
\text { crosslinking hydrogels }\end{array}$ & $\begin{array}{l}\text { Open; } \\
\text { Intramyocardial }\end{array}$ \\
\hline $\begin{array}{l}\text { Spaulding, } \\
\text { K.A et al. } \\
2020 \text { [27] } \\
\end{array}$ & $\begin{array}{l}\text { Left thoracotomy with } \\
\text { suture ligation }\end{array}$ & $\begin{array}{l}\text { LAD and its } \\
\text { diagonal branches }\end{array}$ & Acellular & $\begin{array}{l}\text { NIPAAm-PEG1500 hydrogel } \\
\text { (Sigma-Aldrich, USA) }\end{array}$ & $\begin{array}{l}\text { Open; } \\
\text { Intramyocardial }\end{array}$ \\
\hline $\begin{array}{l}\text { Takehara, N } \\
\text { et al. } 2008 \\
{[28]}\end{array}$ & $\begin{array}{l}90 \mathrm{~min} \text { intracoronary } \\
\text { balloon occlusion, }\end{array}$ & LAD & $\begin{array}{l}\text { bFGF (Kaken } \\
\text { Pharmaceutical } \\
\text { Co., Tokyo, Japan) }\end{array}$ & Gelatin hydrogel & $\begin{array}{l}\text { Open; } \\
\text { Intramyocardial }\end{array}$ \\
\hline
\end{tabular}


followed by

reperfusion

\begin{tabular}{|c|c|c|c|c|c|}
\hline $\begin{array}{l}\text { Thang Duc } \\
\text { Vu et al. } \\
2011 \text { [29] }\end{array}$ & $\begin{array}{l}\text { Left thoracotomy with } \\
\text { suture ligation }\end{array}$ & Proximal LCx & Platelet-rich plasma & $\begin{array}{l}\text { Hyaluronate Gelatin (Glycosan } \\
\text { BioSystems Inc, Salt Lake City, UT, } \\
\text { USA) }\end{array}$ & $\begin{array}{l}\text { Open; } \\
\text { Intramyocardial }\end{array}$ \\
\hline $\begin{array}{l}\text { Jay H, } \\
\text { Traverse et } \\
\text { al. } 2019 \text { [30] }\end{array}$ & $\begin{array}{l}\text { Patients with } 1 \text { st } \\
\text { STEMI treated by PCI } \\
\text { within past } 60 \text { days to } \\
3 \text { years with moderate } \\
\text { LV dysfunction. }\end{array}$ & - & Acellular & $\begin{array}{l}\text { VentriGelTM - ECM from } \\
\text { decellularized porcine } \\
\text { myocardium }\end{array}$ & $\begin{array}{l}\text { Transcatheter } \\
\text { delivery through } \\
\text { endocardium into } \\
\text { myocardium }\end{array}$ \\
\hline
\end{tabular}

\subsection{Less invasive administration modes}

One of the most important aspects of hydrogel-based myocardial restoration therapy is the mode of delivery. In the context of increasing role of minimally invasive techniques, a particular emphasis has been placed on shifting away from open heart surgery to catheter-based techniques. We doubt that any restoration method involving major surgical trauma can survive as stand-alone treatment, as no patient, cardiologist or surgeon will adopt it. Second, therapy may have to be chronic and repeated, i.e. multiple sessions in the process of time post-MI, as HF chronifies. The patient cannot undergo countless re-dos if the procedure is invasive. This has prompted researchers to develop new devices for pinpoint delivery of therapeutic compounds into a desired area of myocardium. As a result, catheter-based techniques for myocardial restoration therapy have evolved from simple intracoronary injections (which is far from perfect due to rapid wash out of an intravascular compound) to techniques with a more efficient therapeutics' retention. One of the examples is the TransAccess catheter system with a fluoroscopic and intravascular ultrasound guidance which was used for autologous skeletal myoblasts delivery [35]. Currently, the most advanced device for intramyocardial delivery of therapeutic compounds is the NOGA system (Figure 3). The latter allows to perform three-dimensional electromechanical mapping of LV in order to identify target zones and perform precise transendocardial injections of therapeutics. Available data and the authors' own experience derived from large animal models confirms that the NOGA device is safe and highly effective.
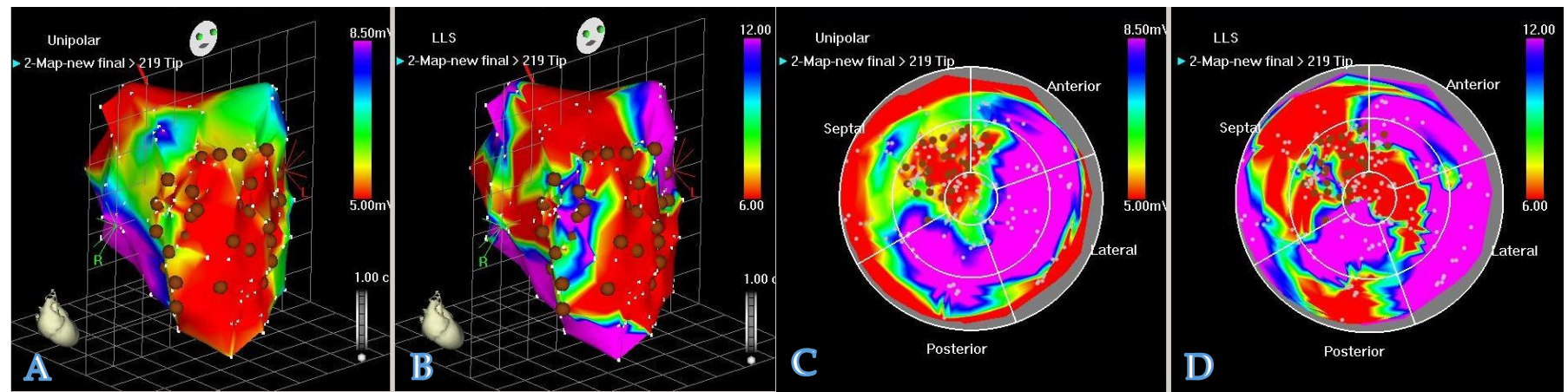

Figure 3. NOGA ${ }^{\circledR}$ system allows to visualize the LV-contraction in 3D. NOGA able to map the heart in full $360^{\circ}$ rotation. A,C: Unipolar voltage map $(\mathrm{mV})$ and B,D: Regional wall motion map by local linear shortening (LLS\%) can help the electromechanical assessment of the myocardium. NOGA shows Viability on the left column, Dense scar is visible at the apex and the antero-septal wall (Red), Scar area $(<0.5 \mathrm{mV})=$ RED; Viable tissue $(>1.5 \mathrm{mV})=$ PURPLE. Comparing the bipolar and unipolar maps, NOGA able to define border-zone areas better.

\section{Conclusions}

Less Invasive procedures, coupled with injectable compounds present a valid platform for a translational restoration protocol, which may be adopted by Interventional Cardiologists and Heart Surgeons. Polytherapeutic adjuvants, such as antioxidants, paracrine-active drugs, antiinflammatory substances, may be added to the protocls, to ensure sustained myocardial restoration effect. 
As discussed in the present paper, among all biomaterials currently used in cardiac tissue engineering, injectable hydrogels with their potential for minimally invasive delivery, in-vivo breakdown into harmless derivatives, represent the most promising therapeutic option. However, the translational pathway from bench to bedside is challenging and still needs to be explored. It can be anticipated that in the next decades the role of cell-vehicle compounds in the treatment of ischemic HF patients will expand and injectable hydrogels will penetrate into the clinical arena to a higher extent.

Supplementary Materials: The following are available online at www.mdpi.com/xxx/s1, Figure S1

Author Contributions: Conceptualization, M.K.; F.S. and T.K.; methodology, E.L., F.S., W.W.; software, E.L., W.W.; validation, F.S., M.K.; formal analysis, W.W., E.L., F.S.; resources, F.S., M.K.; data curation, F.S., E.L., W.W.; writing - original draft preparation, M.K., E.L., W.W.; writing-review and editing, F.S., T.K.; visualization, W.W.; supervision, T.K.; project administration, F.S.; funding acquisition, T.K. All authors have read and agreed to the published version of the manuscript.

Funding: This research was funded by National Research, Innovation and Enterprise (NMRC), Singapore, Centre Grant (CG) programme, CGAug16M008 [SCEPTRE grant] and The APC was funded by the same.

\section{Acknowledgments: None}

Conflicts of Interest: The authors declare no conflict of interest. The funders had no role in the design of the study; in the collection, analyses, or interpretation of data; in the writing of the manuscript, or in the decision to publish the results.

Institutional Review Board Statement: Not applicable.

Informed Consent Statement: Not applicable.

Data Availability Statement: Not applicable. 
17. Contessotto1, P.; Orbanić, D. Elastin-like recombinamers-based hydrogel modulates post-ischemic remodeling in a nontransmural myocardial infarction in sheep. Sci. Transl. Med. 2021, 13, eaaz5380.

18. Li, Y.; Chen, X. Injectable hydrogel with MSNs/microRNA-21-5p delivery enables both immunomodification and enhanced angiogenesis for myocardial infarction therapy in pigs. Sci Adv. 2021, 7(9), eabd6740.

19. Purcell, B.P.; Barlow, S.C. Delivery of a matrix metalloproteinase-responsive hydrogel releasing TIMP-3 after myocardial infarction: effects on left ventricular remodeling. Am J Physiol Heart Circ Physiol. 2018, 315, H814-H825.

20. Chang, M.Y.; Huang T.T. Injection of Human Cord Blood Cells With Hyaluronan Improves Postinfarction Cardiac Repair in Pigs. Stem Cells Transl Med. 2016, 5(1), 56-66.

21. Ifkovits, J.L.; Tous, E. Injectable hydrogel properties influence infarct expansion and extent of postinfarction left ventricular remodeling in an ovine model. Proc Natl Acad Sci U S A. 2010, 107(25), 11507-12.

22. Koudstaal, S.; Bastings, M.M.C. Sustained Delivery of Insulin-Like Growth Factor-1/Hepatocyte Growth Factor Stimulates Endogenous Cardiac Repair in the Chronic Infarcted Pig Heart. J. of Cardiovasc. Trans. Res. 2014, 7, $232-241$.

23. Li, Y.D.; Chang, M.Y. Injection of Peptide nanogels preserves postinfarct diastolic function and prolongs efficacy of cell therapy in pigs. Tissue Eng Part A. 2015, 21(9-10), 1662-71.

24. Liu, Y.; Sun, L. Application of bFGF and BDNF to Improve Angiogenesis and Cardiac Function. J Surg Res. 2006, 136(1), 8591.

25. Chang, M.Y.; Chang, C.H. The time window for therapy with peptide nanofibers combined with autologous bone marrow cells in pigs after acute myocardial infarction. PLoS One. 2015, 10(3), e0115430.

26. Rodell, C.B.; Lee, M.E. Injectable Shear-Thinning Hydrogels for Minimally Invasive Delivery to Infarcted Myocardium to Limit Left-Ventricular Remodeling. Circ Cardiovasc Interv. 2016, 9(10), e004058.

27. Spaulding, K.A.; Zhu, Y. Myocardial injection of a thermoresponsive hydrogel with reactive oxygen species scavenger properties improves border zone contractility. J Biomed Mater Res A. 2020, 108(8), 1736-1746.

28. Takehara, N.; Tsutsumi, Y. Controlled Delivery of Basic Fibroblast Growth Factor Promotes Human Cardiosphere-Derived Cell Engraftment to Enhance Cardiac Repair for Chronic Myocardial Infarction. J Am Coll Cardiol. 2008, 52, $1858-65$.

29. Vu, T.D.; Pal S.N. An autologous platelet-rich plasma hydrogel compound restores left ventricular structure, function and ameliorates adverse remodeling in a minimally invasive large animal myocardial restoration model: a translational approach: Vu and Pal "Myocardial Repair: PRP, Hydrogel and Supplements". Biomaterials. 2015, 45, 27-35.

30. Traverse, J.H.; Henry, T.D. First-in-Man Study of a Cardiac Extracellular Matrix Hydrogel in Early and Late Myocardial Infarction Patients. Am Coll Cardiol Basic Trans Science. 2019, 4(6), 659-69.

31. Ezekowitz, J.A.; Kaul, P. Declining in-hospital mortality and increasing heart failure incidence in elderly patients with first myocardial infarction. J Am Coll Cardiol. 2009, 53(1), 13-20.

32. Barasa, A.; Schaufelberger, M. Heart failure in young adults: 20-year trends in hospitalization, aetiology, and case fatality in Sweden. Eur Heart J. 2014, 35(1), 5-32.

33. Mamas, M.A.; Sperrin, M. Do patients have worse outcomes in heart failure than in cancer? A primary care-based cohort study with 10-year follow-up in Scotland. Eur J Heart Fail. 2017, 19(9), 1095-1104.

34. Stewart, S.; Ekman, I. Population impact of heart failure and the most common forms of cancer: a study of 1162309 hospital cases in Sweden (1988 to 2004). Circ Cardiovasc Qual Outcomes. 2010, 3(6), 573-80.

35. Siminiak, T.; Fiszer, D. Percutaneous trans-coronary-venous transplantation of autologous skeletal myoblasts in the treatment of post-infarction myocardial contractility impairment: the POZNAN trial. Eur Heart J. 2005, 26, 1188-1195.

\section{List of abbreviations:}

CAC: Circulating Angiogenic Cells; hUMSC: Human Umbilical Mesenchymal Stem Cells; AGHM: Acidic Gelatin Hydrogel Microspheres; bFGF: Basic Fibroblast Growth Factor; MI: Myocardial Infarction; NS: Normal saline; CH: Chitosan Hydrogel; GF: Growth Factor; HG: Hydrogel; NRG: Neuregulin; PBS: Phosphate-buffered Saline; ELRs: Elastin-like recombinamers; HAMMPS: Matrix metalloproteinase-sensitive Hyaluronic acid gel; rTIMP-3: Tissue inhibitors of metalloproteinase (TIMP)3 recombinant protein; CB-MNC: Cord blood ; HA: Hyaluronic acid; MeHA: Methacrylated Hyaluronic acid; NF: Nanofibres; MNC: Mononuclear cells; GF: Growth factor: Insulin-like growth factor 1 (IGF-1) or hepatocyte growth factor (HGF); MNC: Mononuclear cells; BDNF: Brain-derived neurotrophic factor; GH: Guest-Host Hydrogel; DC: Dual-crosslinking hydrogel; hBMCs: Human bone marrow derived mesenchymal cells; hCDCs: human cardiosphere-derived cells; PRP: Plateletrich plasma; AA: Ascorbic acid; STEMI: ST-segment elevated myocardial infraction; PCI: Percutaneous coronary intervention; LV: Left Ventricle; LVEF: Left ventricular ejection fraction; LCx: left circumflex artery; CACs: circulating angiogenic cells; LAD: left anterior descending; NIPAAm: N-isopropyl acrylamide; HEMA: hydroyethyl(methacrylate); MAPLA: methacrylate polylactide; hUMSC: human umbilical mesenchymal stem cells; bFGF: basic fibroblast growth factor; AGHM: acidic gelatin hydrogel microspheres; VEGF: vascular endothelial growth factor; NRG: neurogulin; HA: hyaluronic acid; ELRs: elastin-like recombinamers; MSN: Mesoporous silica nanoparticles; miR: microRNA; rTIMP-3: Tissue inhibitors of metalloproteinase recombinant protein; MMP: matrix metalloproteinase; CB-MNC - cord blood mononuclear cells; MeHA: methacrylated hyaluronic acid; IFG-1: insulin-like growth factor 1; HGF: hepatocyte growth factor;UPy: Ureidopyrimidinone; MNC: mononuclear cells: bFGF: basic fibroblast growth factor; BNDF: brain-derived neurotrophic factor; PEG: Polyethylene glycol; ECM: extracellular matrix; $+\mathrm{dp} / \mathrm{dt}$ : measure of systolic function; -dp/dt: measure of diastolic function; AE: arterial elastance; BV: blood vessel; CI: cardiac index = SV x (heart rate)/BSA/10; CO: cardiac output; ESPVR: end-systolic pressurevolume relationship; IVS: interventricular septum; EDV: end-diastolic volume; EDP: end diastolic pressure; Emax: 
maximum chamber elasticity; FAC: fractional area change; LV EDd: left ventricular end-diastolic dimension; LV ESV: left ventricular end-systolic volume; FAS: fractional area shortening; LVEF: left ventricular ejection fraction; LVEDP: left ventricular end diastolic pressure; MBF: myocardial blood flow; MFR: myocardial flow reserve; MLWHFQ: Minnesota Living with Heart Failure Questionnaire; NYHA: New York Heart Association; PRSW: preload recruitable stroke work; PSV: peak of systolic velocity; SV: stroke volume; WMSI: Wall Motion Score Index; EC: endothelial cell; LVMV: left ventricle mass volume; FL-MMP-2: full length matrix metalloproteinase 2; ROS: reactive oxygen species; BZ: borderzone; LV: left ventricule

171 